\title{
Bienestar psicológico de jóvenes víctimas del conflicto armado ${ }^{1}$
}

\section{Psychological wellbeing of young victims of the armed}

DOI: http://dx.doi.org/10.17981/cultedusoc.11.2.2020.01

Recibido: 14 de enero de 2020 Aceptado: 06 de mayo de 2020 Publicado: 09 de julio de 2020

\author{
Claudina Esther Anaya García \\ Centro Cultural Jáfana Jáfana. Santa Marta (Colombia) \\ claudinis_angar@yahoo.es \\ Linda Carolina Sánchez Rende \\ Fundación Rehabilitación Integral. Santa Marta (Colombia) \\ lisaren-13@hotmail.com
}

\author{
Amanda Miguel Iguarán Jiménez \\ Universidad del Magdalena. Santa Marta (Colombia) \\ amanjosemiguel0222@hotmail.com
}

Para citar este artículo:

Anaya, C., Sánchez, L. e Iguarán, A. (2020). Bienestar psicológico de jóvenes víctimas del conflicto armado. Cultura, Educación y Sociedad, 11(2). 9-26. DOI: http://dx.doi.org/10.17981/cultedusoc.11.2.2020.01

\section{Resumen}

El bienestar psicológico se entiende como un constructo que tiene dimensiones psicológicas, subjetivas, sociales y comportamentales, relacionados con la salud mental que conducen a las personas a operar de una manera positiva, centrando su atención en el desarrollo de capacidades y crecimiento personal para hallar satisfacción en la vida. El Objetivo es determinar el bienestar psicológico y su relación con algunas variables socio demográficas en jóvenes víctimas del conflicto armado registrado en la Unidad de Víctimas de Santa Marta, Colombia. Mediante un enfoque cuantitativo, con un tipo de investigación empírico analítico -correlacional y un diseño transversal, se empleó el estadístico Chi cuadrado y se aplicó la Escala de Bienestar Psicológico de Ryff a una muestra no probabilística intencional de ciento quince (115) jóvenes víctimas, entre las edades de 18 a 22 años. Los resultados obtenidos indicaron la relación de un bienestar psicológico y el nivel de estudios en un 0,010. No obstante, no se halló incidencia en las variables de edad, sexo, vinculación laboral e ingreso económico con el bienestar psicológico. En cuanto a las dimensiones existe una significancia de 0,042 entre el sexo y relaciones positivas; de 0,005 entre nivel de estudios y autoaceptación; 0,048 entre ingresos económicos y relaciones positivas y de 0,016 entre la edad y dominio del entorno. En conclusión, el bienestar psicológico de jóvenes víctimas del conflicto armado está relacionado con el crecimiento personal y el académico.

Palabras clave: Bienestar psicológico; víctima; jóvenes; conflicto armado

\section{Abstract}

Psychological well-being is understood as a construct that has psychological, subjective, social and behavioral dimensions related to mental health that lead people to operate in a positive way, focusing their attention on the development of capacities and personal growth to find satisfaction. in the life. The objective is to determine the psychological wellbeing and its relationship with some socio-demographic variables in young victims of the armed conflict registered in the Victims Unit of Santa Marta, Colombia. Using a quantitative approach, with a type of empirical analyticalcorrelational research and a cross-sectional design, the Chisquare statistic was used and the Ryff Psychological WellBeing Scale was applied to an intentional non-probability sample of one hundred and fifteen (115) young victims, between the ages of 18 to 22 years. The results obtained indicated the relationship between psychological well-being and educational level at 0.010 . However, no incidence was found in the variables of age, sex, employment relationship and economic income with psychological well-being. Regarding the dimensions, there is a significance of 0.042 between sex and positive relationships; 0.005 between educational level and self-acceptance; 0.048 between economic income and positive relationships and 0.016 between age and domain of the environment. In conclusion, the psychological well-being of young victims of the armed conflict is related to personal and academic growth.

Keywords: Psychological wellbeing; victim; youth; armed conflict

${ }^{1}$ Producto de la investigación desarrollada durante el periodo de un año, ejecutada desde la maestría en Desarrollo Integral de Niños y Adolescentes de la Universidad Cooperativa de Colombia-UCC (Santa Marta, Colombia). 


\section{INTRODUCCIÓN}

En Colombia se ha desarrollado un conflicto armado interno de tipo no convencional y con una baja intensidad, que poco a poco ha ido tomando dimensiones regionales complejas, cuyos orígenes se han visto en controversia por las ideologías políticas y por los problemas agrarios que no se han resuelto (Trejos, 2013). Este conflicto ha causado múltiples daños a la sociedad colombiana, daños no sólo medibles con el número de muertes de civiles y la destrucción material, también un impacto intangible como la afectación del proyecto de vida de miles de personas y familias, cortando las posibilidades de futuro a una parte de la sociedad y agrietando el desarrollo democrático. El conflicto armado produce daños emocionales y psicológicos, morales, políticos y socioculturales, siendo principalmente los niños, niñas y adolescentes los más indefensos y vulnerables ante tales estragos (República de Colombia. Centro Nacional de Memoria Histórica, 2013).

El conflicto armado es definido como el que no está cubierto por el artículo 1 del Protocolo adicional a los convenios de Ginebra del 12 de agosto de 1949, relativo a la protección de las víctimas de los conflictos armados internacionales (Protocolo I) y que se desarrollen en el territorio de una alta parte contratante, entre sus fuerzas armadas y fuerzas armadas disidentes o grupos armados organizados que bajo la dirección de un mando responsable, ejerzan sobre una parte de dicho territorio un control tal que les permita realizar operaciones militares sostenidas y concertadas y aplicar el presente protocolo, es decir que el conflicto armado es aquel conflicto no internacional que viola el convenio de Ginebra (Comité Internacional de la Cruz Roja, CICR, 2005).

Una víctima es la persona que, individual o colectivamente, como resultado de actos $\mathrm{u}$ omisiones que violan las normas internacionales de derechos humanos o el derecho internacional humanitario, haya sufrido daños, inclusive lesiones físicas o mentales, sufrimiento emocional, pérdida financiera o menoscabo sustancial de sus derechos fundamentales. Se podrá considerar también "víctimas" a los miembros de la familia directa o personas a cargo de la víctima directa, así como a las personas que, al intervenir para asistir a la víctima o impedir que se produzcan otras violaciones, hayan sufrido daños físicos, mentales o económicos. La condición de una persona como "víctima" no debería depender de que se haya identificado, capturado, enjuiciado o condenado al autor de la violación, y debería ser independiente de toda relación que pueda existir o haber existido entre la víctima y ese autor (Muñoz y Orrego, 2016).

Para la Unidad de Víctimas de Colombia la cifra de niños y adolescentes víctimas del conflicto armado asciende a 2.500.000 (Semana, 2016). Los niños, niñas y adolescentes además de ser desplazados, asesinados o desaparecidos, también han sido víctimas de otros delitos atroces. El Grupo de Memoria Histórica, describe que en las regiones del Caribe como en el Magdalena, Córdoba y Bolívar, las niñas han sido más víctimas de violaciones sexuales que en las otras regiones del país y que la mayor parte de esos casos han ocurrido en inmediaciones de la Sierra Nevada de Santa Marta, donde había campeado durante muchos años la guerrilla y los paramilitares (Gossaín, 2015).

Respecto a esto, Goleman (2004) expone que los niños y niñas en situación de desplazamiento forzado son los más vulnerables de los vulnerados, tomando en cuenta que los actos violentos son más dañinos que las catástrofes naturales (...) porque las víctimas de 
la violencia sienten que han sido intencionalmente seleccionados como blancos de maldad (p. 237), la violencia a la que se refiere es "aquella que tiene la intencionalidad de producir daño mediante la fuerza y abuso de poder para afectar las capacidades individuales y colectivas"(Gómez, 2006, p. 16); esta configura traumas que a través del anclaje a experiencias dolorosas, determinan reacciones desajustadas y emociones con tendencia a la ambigüedad, la depresión o hacia la futilidad.

Por consiguiente; según Ramírez et al (2016) las afectaciones del conflicto armado van desde un deterioro en la salud mental hasta un reordenamiento de los estilos de vida tanto individual como comunitario. Y es que la violencia ha afectado a tantas personas $\mathrm{y}$ ha arrebatado vidas que han terminado impactando las estructuras familiares y comunitarias, y con ésta los contextos de socialización, generando secuelas a nivel individual y social (Agencia Canadiense de Desarrollo Internacional, ACDI, 2009). Ante lo expuesto las afectaciones psicológicas que se pueden producir a raíz del conflicto armado en los menores, pueden tener incidencia en su bienestar psicológico y en distintas dimensiones de la vida.

El Bienestar psicológico se entiende como un constructo que tiene dimensiones psicológicas, subjetivas, sociales y comportamientos relacionados con la salud mental que conducen a las personas a operar de una manera positiva, centrando su atención en el desarrollo de capacidades y crecimiento personal para hallar satisfacción en la vida (Díaz, et al., 2006). Ryff y Keyes (1995) consideran que el ser humano está en busca de perfeccionarse y de cumplir con su potencial para lo cual esto le sirve para tener un propósito de vida que le ayude a darle un significado. Esto implica que el ser humano deba asumir retos y realizar esfuerzos para superarlos y alcanzarlos. Por ello estos autores centran su atención en el desarrollo de las capacidades y del crecimiento personal, concibiéndolas a ambas como los principales indicadores del funcionamiento positivo del individuo (Ryff, 1989a; Ryff \& Keyes, 1995). Planteando con esto los autores un modelo multidimensional de bienestar psicológico compuesto por seis dimensiones: autoaceptación, relaciones positivas con otras personas, autonomía, dominio del entorno, propósito en la vida, y crecimiento personal.

\section{Modelo MUltidimensional DE BIENESTAR PSICOLÓGICO: REFERENTES TEÓRICOS}

Ryff y Keyes (1995) plantearon un modelo multidimensional de bienestar psicológico dividido en seis dimensiones: autoaceptación, relaciones positivas con otras personas, autonomía, dominio del entorno, propósito en la vida, y crecimiento personal, que a continuación se describen:

La auto-aceptación, es uno de los criterios centrales del bienestar. Las personas intentan sentirse bien consigo mismas incluso siendo conscientes de sus propias limitaciones. Teneractitudes positivas hacia uno mismo es una característica fundamental del funcionamiento psicológico positivo. La autoaceptación también implica la capacidad que tiene la persona de sentirse bien con respecto a las consecuencias del pasado, se sienten seguras y capaces de enfrentar cualquiera situación (Castro, 2002). Relaciones positivas con otras personas, capacidad de mantener relaciones sociales basadas en la confianza mutua y empatía (Ryff, 1989b). Las personas autorrealizadas, sienten afecto por los demás y son capaces de brindar amor, establecer fuertes lazos de amistad y se identifican más con los otros (Ryff, 1989b). 
Autonomía, hace referencia a la autodeterminación, independencia y regulación de la conducta. Las personas con autonomía son capaces de resistir en mayor medida la presión social y auto-regulan mejor su comportamiento. De acuerdo a Schwartz (2000), cada persona se autodetermina, en otras palabras, tiene la libertad de decidir qué quiere ser, y luchar por las cosas que les interesa. En efecto, la autonomía es la capacidad que tiene el ser humano para mostrarse ante sí mismo y ante los demás como un ser propositivo, que comprende y participa activamente en el mundo que le rodea, que evalúa las alternativas de acción más ajustadas al contexto, en función de sus intereses y motivaciones (Blanco y Valera, 2007). El dominio del entorno, es la habilidad que tienen las personas para elegir o crear entornos favorables para satisfacer los deseos y necesidades propias. Las personas con un alto dominio del entorno poseen una mayor sensación de control sobre el mundo y se sienten capaces de influir sobre el contexto que les rodea. Propósito en la vida, las personas necesitan tener metas y objetivos que permiten dotar su vida de cierto sentido. Esto no solo aparece en ausencia de dificultades, sino que puedo darse ante situaciones críticas que cuestionan la existencia (Ryff \& Singer, 1998). Y Crecimiento personal, esta dimensión se entiende como el interés que cada individuo tiene por desarrollar sus potencialidades, por seguir creciendo como persona y llevar al máximo las propias capacidades (Ryff, 1989a; Díaz et al., 2006).

Además, el Fondo de las Naciones Unidas para la Infancia (UNICEF, 2004) expone que una significativa estimulación del individuo favorece el desarrollo de habilidades y mejor uso de las funciones ejecutivas, haciendo hincapié en que las habilidades intelectuales y sociales tienen sus raíces en las experiencias vividas durante la primera infancia en el seno familiar. En un hogar donde se respira un ambiente de cariño, de respeto, de confianza y de estabilidad, los niños o niñas se crían y se desarrollan psíquicamente más sanos y seguros, y se relacionarán con el exterior de esta misma forma, con una actitud más positiva y constructiva hacia la vida.

Por otra parte, ante de las afectaciones del conflicto armado a las víctimas que son aproximadamente 8’347.566 (Torres, Rojas y Río, 2018), el estado colombiano en respuesta al restablecimiento de derechos humanos diseñó la ley 1448 (República de Colombia, Congreso de la República, 2011) que reconoce como víctimas a las personas que, individual o colectivamente hayan sufrido un daño por hechos ocurridos desde el 1 de enero de 1985, a consecuencia de infracciones enmarcadas en el Derecho Internacional Humanitario o de violaciones graves y manifiestas a las normas internacionales de los Derechos Humanos, que ocurrieran con ocasión del conflicto armado interno, y que por ello bajo la medida de asistencia, atención y reparación integral a las víctimas del conflicto armado se les repararía su calidad de vida.

\section{Metodología}

\section{Diseño}

La presente investigación es de corte cuantitativo, se realizó la recolección de datos con base a la medición numérica y al análisis estadístico, para establecer patrones de comportamientos y poder correlacionarlos (Hernández, Fernández y Baptista, 2010; Behar, 2008). El diseño es trasversal de tipo correlacional, puesto que buscó medir la diferencia del nivel de bienestar psicológico y las variables socio-demográficas sexo, edad, nivel de escolaridad, condición laboral e ingresos económicos. 


\section{Participantes}

La población de este estudio estuvo conformada por jóvenes víctimas del conflicto armado registrados en el Programa Jóvenes en Acción (Departamento para la Prosperidad Social, DPS, 1969) con un total de 400; de la cual se tomó una muestra intencional de 115 jóvenes menores de 22 años. Los criterios de inclusión tomados en cuenta para la ejecución de esta investigación fueron incluir personas entre los 18 hasta los 22 años de edad y firmar el consentimiento informado, responder la escala de bienestar psicológico y el cuestionario de datos socio demográficos, estar registrados en el Programa Jóvenes en Acción y los criterios de exclusión fueron, no estar registrado en el Programa Jóvenes en Acción, tener más de 22 años de edad y no firmar el consentimiento informado.

A continuación, se presenta la muestra distribuida según sexo, edad, nivel de estudios, condición laboral e ingresos económicos (Tabla 1).

TABLA 1.

Datos sociodemográficos de las jóvenes víctimas del conflicto armado

\begin{tabular}{|c|c|c|}
\hline Sexo & Frecuencia & Porcentaje \\
\hline Masculino & 36 & $31 \%$ \\
\hline Femenino & 79 & $69 \%$ \\
\hline Total & 115 & $100 \%$ \\
\hline \multicolumn{3}{|l|}{ Edad } \\
\hline $18-20$ & 55 & $48 \%$ \\
\hline $21-22$ & 60 & $52 \%$ \\
\hline Total & 115 & $100 \%$ \\
\hline Nivel de estudios & Frecuencia & Porcentaje \\
\hline Primaria & 3 & $3 \%$ \\
\hline Bachillerato & 65 & $57 \%$ \\
\hline Técnico & 14 & $12 \%$ \\
\hline Tecnólogo & 1 & $0.8 \%$ \\
\hline Universidad & 32 & $28 \%$ \\
\hline Total & 115 & $100 \%$ \\
\hline Condición laboral & Frecuencia & Porcentaje \\
\hline Desempleado & 73 & $63 \%$ \\
\hline Independiente & 8 & $7 \%$ \\
\hline Informal & 11 & $10 \%$ \\
\hline Hogar & 9 & $8 \%$ \\
\hline Empleo formal & 14 & $12 \%$ \\
\hline Total & 115 & $100 \%$ \\
\hline Ingreso económico & Frecuencia & Porcentaje \\
\hline Menos de 1 salario & 81 & 70 \\
\hline De 1 a 2 salarios & 32 & 28 \\
\hline Más de 3 salarios & 2 & 2 \\
\hline Total & 115 & 100 \\
\hline
\end{tabular}

Fuente: Elaboración propia. 
En la Tabla 1 se observa cómo se encuentra dividida la muestra por sexo, edad, nivel de estudio condición laboral e ingreso económico. El 31\% es masculino y el 69\% es femenino; siendo un $48 \%$ de edad de 18 a 22 años y el 52\% de 21 a 22 años. El nivel de estudios de las jóvenes víctimas del conflicto armado; ubicándose con mayor porcentaje el nivel bachiller con el 57\% bachiller y el 28\% universitario. Las jóvenes víctimas según su condición laboral. Se ubican con mayor porcentaje los jóvenes desempleados con un $63 \%$, y $12 \%$ con empleo formal. El 70\% de jóvenes victimas gana menos de un salario mínimo, jóvenes, de 1 a 2 salarios mínimo el $28 \%$, y más de 3 salario mínimo un $2 \%$.

\section{Técnicas o Instrumentos}

Para llevar a cabo la recolección de la información, se aplicó a la muestra seleccionada la Escala de Bienestar Psicológico de Ryff (1989b), adaptada por Van Dierendonck (2004) y traducida al español por Díaz et al. (2006, citado por Véliz, 2012). De acuerdo a Véliz (2012) el ámbito de aplicación es para adolescentes y adultos entre las edades de 17 y los 90 años. En la versión utilizada se conservan las seis sub-escalas originales del test, que son: 1) Auto-aceptación, 6 ítems; 2) Relaciones Positivas, 6 ítems; 3) Autonomía, 8 ítems; 4) Dominio del entorno, 6 ítems; 5) Propósito en la vida, 7 ítems; y 6) Crecimiento Personal, 6 ítems. El total de ítems de la Escala es 39, y su formato de respuesta está compuesto por puntuaciones tipo Likert que van de 1 a 6 , donde 1=Totalmente en Desacuerdo y $6=$ Totalmente de Acuerdo.

Este instrumento presenta consistencia interna (medida con Alfa de Cronbach) de las sub-escalas de Bienestar Psicológico de la versión española propuesta por Van Dierendonk (2004) de: Autoaceptación $=.83$, Relaciones positivas $=.81$, Autonomía $=.73$, Dominio del entorno $=.71$, Propósito en la vida $=.83$ y Crecimiento personal $=.68$. La validez factorial de la escala original se comprobó mediante Análisis Factorial Confirmatorio (Método de extracción: Máxima verosimilitud. Método de rotación: Normalización Oblimin con Kaiser), empleando el programa AMOS 5.0. Comprobando su validez a través de la comparación de seis modelos diferentes (Van Dierendonck, 2004).

\section{Sistematización procedimental}

Se ejecutaron varias fases, la primera fase fue de diagnóstico donde se aplicó el instrumento y en su calificación, intervinieron los siguientes ítems: 2, 4, 5, 8, 9, 13, 15, 20, 22, 25, 26, 27, 29, 30, 33, 34, 36. Y para la interpretación se sumaron los puntajes obtenidos y se interpretaron que, a mayor puntaje, mayor bienestar psicológico del individuo. Además de la anterior prueba, en la siguiente fase se aplicó una ficha de datos socio demográfico, por medio de la cual se recogieron datos de edad, sexo, ingresos económicos, nivel de estudios, condición laboral, grupo familiar, hecho victimizante, tipo de medida recibida de la ley de víctimas y restitución de tierras. 


\section{RESULTADOS}

Para el análisis estadístico de los datos, se realizó la tabla de contingencia entre la variable bienestar psicológico, con sus dimensiones y variables socio demográficas. Además; para determinar las posibles diferencias significativas entre las categorías de las variables se empleó la prueba no paramétrica para una muestra Chi-Cuadrado de Pearson, tomándose como nivel de significancia un $\mathrm{p} \leq 0,05$; partiendo de la hipótesis que el bienestar psicológico y sus dimensiones no tienen relación con las variables sociodemográficas; por lo cual si en la prueba se obtiene un $p>0,000$ sería poco probable esta hipótesis, se rechazaría la independencia de las variables y se acepta su relación (Tabla 2).

TABLA 2.

Chi cuadrado de Pearson entre Bienestar Psicológico y variables sociodemográficas

\begin{tabular}{lll}
\hline \multicolumn{2}{c}{ Bienestar Psicológico } & \\
\hline \multirow{2}{*}{ Edad } & $\mathrm{X}^{2}$ & 3,706 \\
& Sig. asintótica (bilateral) & 0,157 \\
Sexo & $\mathrm{X}^{2}$ & $1.595^{\mathrm{a}}$ \\
& Sig. asintótica (bilateral) & 0.451 \\
Nivel de estudios & $\mathrm{X}^{2}$ & $29.303^{\mathrm{a}}$ \\
& Sig. asintótica (bilateral) & 0.010 \\
Vinculación laboral & $X^{2}$ & $8.280^{\mathrm{a}}$ \\
& Sig. asintótica (bilateral) & 0.407 \\
Ingresos Económicos & $X^{2}$ & $2.701^{\mathrm{a}}$ \\
& Sig. asintótica (bilateral) & 0.845 \\
\hline
\end{tabular}

Nota: Sig. Una $p>0,05$ indica diferencia significativa entre las variables

Fuente: Elaboración propia.

En la Tabla 2, se observa un nivel de significancia de 0,010 entre nivel de estudio y bienestar psicológico; es decir que esta variable tiene implicación en el bienestar psicológico. Sin embargo, no se evidencia diferencia significativa del bienestar psicológico con las variables socio demográficas edad, sexo, vinculación laboral e ingresos económicos.

En la Tabla 3 se observa que existe relación entre el sexo y relaciones positivas de 0,042 de significancia; entre nivel de estudios y la dimensión de autoaceptación de 0,005; y entre ingresos económicos y relaciones positivas de 0,048.

Además; se analiza que la edad y vinculación laboral no presenta ningún tipo de relación con las dimensiones de autoaceptación, relaciones positivas y autonomía. Por tanto; la variable edad y condición laboral no determinan el bienestar en estas dimensiones. 
TABLA 3.

Chi cuadrado de Pearson entre las dimensiones de Autoaceptación, Relaciones positivas y Autonomía, y variables socio-demográficas

\begin{tabular}{lllll}
\hline & & Autoaceptación & \multicolumn{1}{c}{$\begin{array}{c}\text { Relaciones } \\
\text { positivas }\end{array}$} & Autonomía \\
\hline \multirow{2}{*}{ Edad } & $\mathrm{X}^{2}$ & 0,046 & 0,784 & 0,282 \\
& Sig. asintótica (bilateral) & 0,83 & 0,376 & 0,595 \\
Sexo & $\mathrm{X}^{2}$ & $0.447^{\mathrm{a}}$ & $4.141^{\mathrm{a}}$ & $0.880^{\mathrm{a}}$ \\
& Sig. asintótica (bilateral) & 0.504 & 0.042 & 0.348 \\
Nivel de estudios & $\mathrm{X}^{2}$ & $20.416^{\mathrm{a}}$ & $9.436^{\mathrm{a}}$ & $6.136^{\mathrm{a}}$ \\
& Sig. asintótica (bilateral) & 0.005 & 0.223 & 0.524 \\
Vinculación laboral & $\mathrm{X}^{2}$ & $3.345^{\mathrm{a}}$ & $0.588^{\mathrm{a}}$ & $1.827^{\mathrm{a}}$ \\
& Sig. asintótica (bilateral) & 0.502 & 0.964 & 0.768 \\
Ingresos económicos & $X^{2}$ & $0.436^{\mathrm{a}}$ & $7.902^{\mathrm{a}}$ & $2.468^{\mathrm{a}}$ \\
& Sig. asintótica (bilateral) & 0.933 & 0.048 & 0.481 \\
\hline
\end{tabular}

Fuente: Elaboración propia.

En la Tabla 4 la dimensión de dominio del entorno tiene una significancia $(0,016)$ con la edad; por lo tanto, se puede determinar que la edad tiene incidencia en esta dimensión del bienestar psicológico. La variable sexo, nivel de estudios, vinculación laboral e ingresos económicos no presentaron significancia entre las dimensiones dominio del entorno, crecimiento personal y propósito en la vida.

TABLA 4.

Chi cuadrado de Pearson entre las dimensiones de Dominio del entorno, Crecimiento personal y Propósito en la vida, y variables socio-demográficas

\begin{tabular}{llllc}
\hline & & \multicolumn{1}{c}{$\begin{array}{c}\text { Dominio del } \\
\text { entorno }\end{array}$} & $\begin{array}{c}\text { Crecimiento } \\
\text { personal }\end{array}$ & $\begin{array}{c}\text { Propósito en } \\
\text { la vida }\end{array}$ \\
\hline \multirow{2}{*}{ Edad } & $\mathrm{X}^{2}$ & 8.313 & 0 & 0,016 \\
& Sig. asintótica (bilateral) & $\mathbf{. 0 . 0 1 6}$ & 1 & 0,899 \\
Sexo & $\mathrm{X}^{2}$ & $3.996^{\mathrm{a}}$ & $0.431^{\mathrm{a}}$ & 0,194 \\
& Sig. asintótica (bilateral) & 0.136 & 0.511 & 0,659 \\
Nivel de estudios & $\mathrm{X}^{2}$ & $12.912^{\mathrm{a}}$ & $12.171^{\mathrm{a}}$ & 12,895 \\
& Sig. asintótica (bilateral) & 0.533 & 0.095 & 0,075 \\
Vinculación laboral & $\mathrm{X}^{2}$ & $0.985^{\mathrm{a}}$ & $3.210^{\mathrm{a}}$ & 3,397 \\
& Sig. asintótica (bilateral) & 0.998 & 0.523 & 0,494 \\
& $X^{2}$ & $7.263^{\mathrm{a}}$ & $3.625^{\mathrm{a}}$ & 0,81 \\
& Sig. asintótica (bilateral) & 0.297 & 0.305 & 0,847 \\
\hline
\end{tabular}

Fuente: Elaboración propia. 
En la Tabla 5 se observa el $15 \%$ del sexo femenino presentan un alto bienestar psicológico, el $70 \%$ en medio y un $15 \%$ bajo. Respecto al sexo masculino un $8 \%$ bajo, $69 \%$ media y un $22 \%$ alto de nivel de bienestar psicológico; es decir que la mayor parte de jóvenes víctimas se encuentra en medio dentro de los porcentajes del bienestar psicológico. Un 7\% más del sexo femenino se ubica en un nivel bajo en comparación al sexo masculino; e igualmente un 7\% más de sujetos masculinos se ubicó en un nivel alto en relación a los de sexo femenino.

Respecto a la edad, el 17\% de los jóvenes mayores de 21 años y el $9 \%$ de los jóvenes de 18 a 20 años se ubicaron en el nivel bajo de bienestar psicológico. Y un $13 \%$ de las jóvenes víctimas de 18 a 20 años y un $22 \%$ de jóvenes mayores de 21 años presentaron un nivel alto de bienestar.

TABLA 5.

Bienestar Psicológico asociado al sexo y la edad

\begin{tabular}{|c|c|c|c|c|}
\hline Sexo & Bajo & Medio & Alto & Total \\
\hline Masculino & $8 \%$ & $69 \%$ & $22 \%$ & $100 \%$ \\
\hline Femenino & $15 \%$ & $70 \%$ & $15 \%$ & $100 \%$ \\
\hline Edad & Bajo & Medio & Alto & Total \\
\hline De 18 a 20 años & $9 \%$ & $78 \%$ & $13 \%$ & $100 \%$ \\
\hline De 21 años o mas & $17 \%$ & $62 \%$ & $22 \%$ & $100 \%$ \\
\hline
\end{tabular}

Fuente: Elaboración propia.

En la Tabla 6 se observa que un 17\% de jóvenes víctimas del conflicto armado presenta un bienestar psicológico alto, un $70 \%$ en medio y el 13\% bajo. El hecho de que los jóvenes se encuentren en su mayoría en un nivel medio de bienestar puede indicar que están experimentando dificultad en la parte social o personal; que les impide un pleno y completo bienestar psicológico.

En las dimensiones del bienestar psicológico un 83 \% de los jóvenes se ubicaron en un nivel medio en la dimensión de autoaceptación, asimismo un $72 \%$ de los jóvenes se encuentra en un nivel medio en propósito en la vida. Lo que indica que tienen un reconocimiento de sus habilidades y tienen proyectos que esperan alcanzar en un futuro.

En relaciones positivas un $78 \%$ de los jóvenes está en un nivel bajo, el $66 \%$ de tiene un nivel bajo de autonomía y el $66 \%$ de los jóvenes presenta un nivel bajo de dominio del entorno. Lo que quiere decir; que los jóvenes presentan dificultades para mantener relaciones positivas; poco conocimiento de sí mismo, pobre control de los espacios donde interactúan en la adaptación y reconocimiento para detectar los peligros y oportunidades que se puedan encontrar en estos espacios. 
TABla 6.

Nivel de Bienestar Psicológico y sus dimensiones en jóvenes víctimas del conflicto armado.

\begin{tabular}{|c|c|c|}
\hline \multicolumn{3}{|c|}{ Bienestar Psicológico } \\
\hline \multirow{3}{*}{ Bienestar Psicológico } & Alto & $17 \%$ \\
\hline & Medio & $70 \%$ \\
\hline & Bajo & $13 \%$ \\
\hline \multirow{3}{*}{ Autoaceptación } & Alto & $0 \%$ \\
\hline & Medio & $83 \%$ \\
\hline & Bajo & $17 \%$ \\
\hline \multirow{3}{*}{ Relaciones Positivas } & Alto & $0 \%$ \\
\hline & Medio & $22 \%$ \\
\hline & Bajo & $78 \%$ \\
\hline \multirow{3}{*}{ Autonomía } & Alto & $0 \%$ \\
\hline & Medio & $34 \%$ \\
\hline & Bajo & $66 \%$ \\
\hline \multirow{3}{*}{ Dominio del entorno } & Alto & $1 \%$ \\
\hline & Medio & $42 \%$ \\
\hline & Bajo & $57 \%$ \\
\hline \multirow{3}{*}{ Crecimiento personal } & Alto & $0 \%$ \\
\hline & Medio & $40 \%$ \\
\hline & Bajo & $60 \%$ \\
\hline \multirow{3}{*}{ Propósito en la vida } & Alto & $0 \%$ \\
\hline & Medio & $72 \%$ \\
\hline & Bajo & $28 \%$ \\
\hline Total & & $100 \%$ \\
\hline
\end{tabular}

Fuente: Elaboración propia.

\section{DiscUSIÓN}

En la presente investigación correspondiente a relación del nivel de bienestar psicológico y variables socio-demográficas en jóvenes víctimas del conflicto armado registrados en la Unidad de Víctimas de Santa Marta (Colombia) se encontró correlación entre el nivel de estudios y bienestar psicológico determinado por un nivel de significancia de 0,010, por tanto, el acceso a la educación y formación académica favorecen un mayor nivel de bienestar psicológico. Lo antecedente coincide, con uno de los planteamientos centrales de Ryff y Keyes (1995), que el desarrollo de las capacidades y del crecimiento personal son indicadores fundamentales en el funcionamiento positivo de un ser humano. Sin embargo, no se halló incidencia de las variables la edad, sexo, vinculación laboral e ingreso económico con el bienestar psicológico. 
De acuerdo a lo anterior, la variable nivel de estudio presentó una significancia de 0,010 con bienestar psicológico; lo que indica que el nivel de estudio favorece el bienestar. Y con las dimensiones, sólo con auto aceptación se halló una significancia de 0,005. Empero, Rodríguez, Negrón, Maldonado, Quiñones y Toledo (2015) en las diferencias por nivel de estudio hallaron que los promedios del nivel de pregrado y doctorado difieren significativamente (p. <.05), los estudiantes doctorales tienen promedios más altos en relaciones positivas con otros. Asimismo, González y Marrero (2017), analizaron que las personas con estudios universitarios tenían mayor crecimiento personal, relaciones positivas y propósito en la vida que los de bachiller. Por tanto; la formación académica es un aspecto importante que incide en un nivel de bienestar psicológico óptimo. Aunque; hay que tener presente que la población estudiada tiene condición vulnerable; y el 56\% sólo cuenta con bachiller, por lo que se aprecia que el acceso a la educación superior está siendo una limitante en los jóvenes para presentar un nivel de bienestar alto. Incluso, Morales y González (2014) afirman que, en los estudiantes con un alto índice de vulnerabilidad, su proyecto de vida se torna inestable, impredecible e inseguro afectando esto a su vez el bienestar psicológico.

Respecto al sexo y bienestar psicológico no se presentó diferencia significativa; es decir en las puntuaciones de los niveles de bienestar entre hombres y mujeres no hubo distinción relevante. Lo anterior; indica que en esta investigación el sexo no tuvo influencia en el bienestar, ser hombre o mujer no tiene ninguna implicación en el nivel del bienestar psicológico que tenga un joven. De igual manera, González, Montoya, Casullo y Bernabéu (2002) en su estudio no hallaron relación alguna entre el bienestar psicológico y el sexo, ya que el Índice de Bienestar Psicológico General fue semejante en ambos sexos; aunque los varones se destacaron significativamente en la aceptación de sí mismos. Pero, Viñas, González, García, Malo y Casas (2015), encontraron un menor bienestar personal en las mujeres, el cual atribuyeron a una combinación de factores psicosociales, y cambios hormonales. Pese a esto, Matalinares et al. (2016), comprobaron que las mujeres en la escala general perciben un mayor bienestar psicológico a diferencia de los varones.

En este orden de ideas, específicamente con las dimensiones, se presentó una diferencia significativa de 0,042 del sexo con relaciones positivas. No obstante, Rodríguez, Negrón, Maldonado, Quiñones y Toledo (2015) hallaron diferencias por sexo entre hombres y mujeres; encontrando que las mujeres exhiben niveles más altos de auto aceptación $(\mathrm{M}=19.96, \mathrm{SD}=3.50)$, dominio del entorno $(\mathrm{M}=22.92$, $\mathrm{SD}=4.12)$, crecimiento personal $(\mathrm{M}=21.27, \mathrm{SD}=4.12), \mathrm{t}(762)=-1.93$. p. <.05) y propósito en la vida t $(762)=2.24$, p. <.02). Mientras, los hombres exhiben niveles más altos en relaciones positivas con otros $(\mathrm{M}=23.61, \mathrm{SD}=4.60)$ y autonomía $(\mathrm{M}=27.14, \mathrm{SD}=4.60)$. Matalinares et al. (2016) encontraron que las mujeres perciben mayor crecimiento personal y propósito de vida. Además, los varones manifiestan mayor aceptación de sí mismos que las mujeres. Asimismo, en el estudio de Barcelata y Rivas (2016), los hombres reportaron mayor autoaceptación $(\mathrm{F}=9.840, \mathrm{p}=.002, \mathrm{gl}=1)$ y relaciones positivas con otros $(\mathrm{F}=6.755$, $\mathrm{p}=.010, \mathrm{gl}=1$ ). De igual manera, Romero et al. (2010) y Mayordomo, Sales, Satorres y Meléndez (2016) encontraron que los hombres puntuaron más alto en las escalas de auto aceptación y de autonomía. Adicionalmente, en el estudio de González y Marrero (2017), las mujeres mostraron mayor relaciones positivas $\mathrm{F}(1,753)=5.93, \mathrm{p}<.05$, 
$\eta 2=.01,1-\beta=.68 ; \mathrm{y}$ los hombres informaron mayor autonomía $\mathrm{F}(1,753)=3.28, \mathrm{p}=.070$, $\eta 2=.01,1-\beta=.44$. Acerca de las distenciones entre el sexo y las dimensiones del bienestar psicológico; García (2013), comenta que las diferencias en función del sexo podrían explicarse por orientaciones axiológicas distintas entre hombres y mujeres.

Entre el bienestar psicológico y la edad no se determinó significancia alguna, sólo se presentó una diferencia de 0,016 de la edad con el dominio del entorno. Igualmente; González, et al. (2002), no encontraron relaciones significativas entre la edad y el bienestar psicológico. Al contrario, Romero et al. (2010) hallaron mayor frecuencia de los jóvenes (13-16 años) en la dimensión autoaceptación, seguidamente en propósito de vida y ésta se encuentra acompañada con igual valor de frecuencia con el crecimiento personal. Asimismo, en el estudio de González y Marrero (2017), los jóvenes (17-30 años) mostraron mayor crecimiento personal $\mathrm{F}(1,752)=3.96, \mathrm{p}<.05, \eta 2=.01,1-\beta=.51$. Del valle, Hormaechea y Urquijo (2015), observaron que la edad promedio de 20 años mostró puntuaciones promedio de Bienestar Psicológico superiores a los de la población normotípica, con una edad promedio de 33 años en todas las escalas; además el tamaño del efecto de las diferencias es grande en relaciones positivas y crecimiento personal, mediano para auto aceptación, dominio del entorno y propósito en la vida y muy pequeño para autonomía.

Mayordomo et al. (2016) comprobaron que en las relaciones positivas con otros y la dimensión del crecimiento personal los jóvenes tuvieron mayores puntuaciones. Pero, en la dimensión de dominio del entorno los adultos puntuaron más alto. Muratori, Zubieta, Ubillos y González (2015) hallaron que la edad se correlaciona directamente con tres de las dimensiones del bienestar psicológico: Autonomía, $r(355)=0,15, p=0,004$, Dominio del Entorno, $r(355)=0,16, p=0,003$, y Propósito en la Vida, $r(355)=0,12, p=0,029$; concluyendo que a mayor edad aumenta la percepción de independencia, control del medio y metas vitales. García (2013) halló que los participantes adultos maduros (30-55 años) puntuaron significativamente más alto que los jóvenes (18-29 años) en autoaceptación, dominio del entorno, crecimiento personal y propósito en la vida.

Atribuyendo estas diferencias a cambios significativos en las tendencias motivacionales a lo largo del ciclo vital. Autores como Mayordomo et al. (2016), plantean en cuanto a las dimensiones del bienestar psicológico que las relaciones positivas con otros y la autoaceptación son estables a lo largo del desarrollo; y dominio del entorno y la autonomía se incrementan en el periodo al finalizar la juventud y llegar al envejecimiento, y propósito en la vida y crecimiento personal presentan un descenso a lo largo del ciclo vital.

En el caso de la variable de ingresos económicos sólo presentó diferencia significativa con la dimensión de relaciones positivas de 0,048. Asimismo, Acosta y Palacios (2008) en su estudio no hallaron ninguna asociación de las dimensiones de bienestar psicológico con ingresos económicos. Lo que podría indicar que la condición económica no está relacionada con las dimensiones del bienestar psicológico o existen pocos estudios que analicen la correlación de estas variables.

Acerca de la variable vinculación laboral no hubo ninguna diferencia significativa con bienestar psicológico, ni con sus dimensiones. Aunque, González y Marrero (2017) en su estudio observaron diferencias significativas en las variables de bienestar psicológico con 
la vinculación laboral; ya que las personas empleadas tenían mayor crecimiento personal y relaciones positivas con otros que las desempleadas. Además, las personas vinculadas laboralmente mostraron mayor dominio del entorno, autoaceptación y propósito en la vida que los desempleados.

Respecto a los datos descriptivos de esta investigación del nivel de bienestar psicológico en las jóvenes víctimas del conflicto, se identificó que el 70\% de la muestra de jóvenes se ubicó en un nivel medio; un $17 \%$ en alto y $13 \%$ en bajo. De igual manera, Matalinares et al. (2016) encontraron resultados similares en estudiantes universitarios en dos ciudades de Perú; ya que en Lima un $16.7 \%$ se ubicó en alto, un $74 \%$ en media y $9.4 \%$ en bajo; y en Huancayo, el 12.8 en alto, $75.5 \%$ en medio y 11.8 en baja.

Asimismo, Yarlequé et al. (2016) hallaron un $66.4 \%$ en medio, un $16.7 \%$ en alto y un $16.9 \%$ en bajo. Por consiguiente; en los jóvenes victimas predominó el nivel medio de bienestar psicológico, dato que se debe analizar teniendo en cuenta las experiencias violentas que padecieron a causa del conflicto armado que generaron afectaciones en este tipo de población. Patiño (2015) dice que los niveles de bienestar están mediados por las condiciones y experiencias de adaptación frente al nuevo contexto de roles y hábitos que pueden percibir difíciles de afrontar.

Manrique, Turizo y Martínez (2008) plantean que las experiencias traumáticas que las personas desplazadas han tenido producto de la violencia les ha dejado secuelas que trascienden el ámbito físico y afectan el bienestar psicológico. Sumado a esto, Oramas, Santana y Vergara (2006) son claros en explicar que el bienestar psicológico de un individuo constituye un indicador positivo de la relación del sujeto consigo mismo y con su medio, que incluye aspectos valorativos y afectivos que se integran en la proyección futura y el sentido de la vida. Aspectos que pueden estar fragmentados en la población de jóvenes a raíz de la violencia.

En relación con el nivel de las dimensiones de bienestar psicológico, en auto aceptación el $83 \%$ y propósito en la vida $72 \%$ de jóvenes víctimas se ubicaron en el nivel medio. En relaciones positivas, autonomía, dominio del entorno y crecimiento personal los jóvenes se ubicaron con mayor porcentaje en el nivel bajo. Frente a esto, Manrique et al. (2008), exponen que por la experiencia traumática en las personas víctimas se afecta la capacidad para entablar relaciones de empatía y confiar en los otros; la valoración sobre sus capacidades personales, el interés en desarrollar sus potencialidades, la seguridad de sus convecciones, además experimentan pensamientos de incapacidad para controlar el mundo y de imposibilidad de generar oportunidades que les permitan satisfacer sus necesidades.

Por último, el bienestar psicológico no se puede analizar alejado de las circunstancias y experiencias de vida de las jóvenes víctimas del conflicto armado. Veenhoven (citado en Morales y González, 2014), expone que el bienestar psicológico está relacionado con el contexto sociocultural y hace énfasis en señalar que los jóvenes que ingresan al sector educativo y cuentan con oportunidades alcanzan un mejor bienestar psicológico. En relación a esto, Domínguez (2018), plantea que la falta de apoyo social e institucional y la experiencia de violencia o maltrato en un joven ocasionan poca adaptación al medio, afectando su capacidad para enfrentar la adversidad y habilidad para identificar el peligro y las oportunidades. 


\section{Conclusiones}

En respuesta al objetivo general de esta investigación, enfocado en determinar la relación del nivel de bienestar psicológico y variables socio-demográficas en jóvenes víctimas del conflicto armado se concluye que no se encontraron diferencias significativas entre las variables la edad, sexo, vinculación laboral e ingreso económico y el bienestar psicológico; sin embargo, si se halló correlación del nivel de estudios con bienestar psicológico. Por tanto, para los jóvenes participantes en este estudio su bienestar psicológico está relacionado con llevar a cabo sus proyectos de vida, es decir acceder a la educación y escalar en el nivel de formación favorece en mayor medida un alto nivel de bienestar. Conforme a lo anterior, se recomienda que en la implementación de las medidas de atención, asistencia y reparación integral con jóvenes victimas también se generen acciones estructurales que favorezcan el desarrollo de capacidades y crecimiento personal de estos para que puedan llevar a cabo su proyecto de vida y esta manera incidir en su grado de bienestar psicológico y calidad de vida.

En referencia a las dimensiones de bienestar psicológico y variables socio demográficas se encontró significancia entre la edad y dominio del entorno, lo que indica que el ciclo vital tiene cierta incidencia en la sensación de control que experimenta el individuo hacia el entorno. También hubo significancia del sexo e ingresos económicos con relaciones positiva, lo que evidencia es este estudio que el sexo y los ingresos económicos influyen en la capacidad de sostener relaciones sociales.

En términos generales haciendo énfasis en los niveles de bienestar psicológico el mayor porcentaje de las jóvenes víctimas del conflicto armado se encuentra en un nivel medio de bienestar; y en las dimensiones se ubicaron en mayor porcentaje en nivel bajo y medio. Resultados que no se toman con sorpresa, ya que los jóvenes y sus familias fueron afectados en su integridad y dignidad por el conflicto armado, es decir que es de esperarse un medio o bajo nivel de bienestar y en sus dimensiones en una población que fue objeto de abuso y vulneración de sus derechos humanos.

\section{REFERENCIAS}

Acosta, C. y Palacios, N. (2008). Situación económica y bienestar psicológico en adultos mayores de Balancán, Tabasco. Horizonte Sanitario, 7(3), 9-19. https://doi.org/10.19136/ hs.a7n3.198

ACDI. (2009). Colombia: Huellas del conflicto en la primera infancia. Bogotá, D.C.: SCC, OEI. Recuperado de https://scp.com.co/wp-content/uploads/2014/08/ColombiaHuellas-del-conflicto-en-la-primera-infancia-Save-the-children.pdf

Barcelata, B. y Rivas, D. (2016). Bienestar psicológico y satisfacción vital en adolescentes mexicanos tempranos y medios. Revista Costarricense de Psicología, 35(2), 119-137. Disponible en http://www.rcps-cr.org/openjournal/index.php/RCPs/article/view/84

Behar, D. (2008). Metodología de la investigación. Bogotá, D.C.: Shalom. Recuperado de http://rdigital.unicv.edu.cv/bitstream/123456789/106/3/Libro\%20metodologia\%20investigacion\%20este.pdf?hlnophdjmglfcbaa 
Blanco, A. y Valera, S. (2007). Los fundamentos de la intervención psicosocial. En: B. Amalio \& J. Rodríguez, Intervención Psicosocial. Madrid: MacGrawhill.

Castro, A. (2002). Investigaciones argentinas sobre el bienestar psicológico. En, M. Casullo (Comp.), Evaluación del bienestar psicológico en Iberoamérica. Buenos Aires: Paidós.

CICR. (2005). Protocolo I adicional a los Convenios de Ginebra de 1949 relativo a la protección de las víctimas de los conflictos armados internacionales, 1977. [Online]. Disponible en https://www.icrc.org/es/document/protocolo-i-adicional-convenios-ginebra1949-proteccion-victimas-conflictos-armados-internacionales-1977

Del Valle, M., Hormaechea, F. y Urquijo, S. (2015). El Bienestar Psicológico: Diferencias según sexo en estudiantes universitarios y diferencias con población general. Revista Argentina de Ciencias del Comportamiento, 7(3) 6-13. Disponible en https://revistas. unc.edu.ar/index.php/racc/article/view/10723

Díaz, D., Rodríguez, R., Blanco, A., Moreno, B., Gallardo, I., Valle, C. y Dierendonck, D. (2006). Adaptación española de las escalas de bienestar psicológico de Ryff. Psicothema, 18(3), 572-577. Recuperado de http://www.psicothema.com/pdf/3255.pdf

Domínguez, L. (2018). La adolescencia y la juventud como etapas del desarrollo de la personalidad distintas concepciones en torno a la determinación de sus límites y regularidades. Boletín Electrónico de Investigación de la Asociación Oaxaqueña de Psicología, 4(1), 69-76. Disponible en https://imbiomed.com.mx/1/1/articulos. php?method=showDetail\&id_revista=166\&id_seccion=3168\&id_ejemplar=5003\&id_ articulo $=49381$

García, J. (2013). Bienestar psicológico, edad y género en universitarios españoles. Salud \& Sociedad, 4(1), 48-58. Recuperado de http://pepsic.bvsalud.org/pdf/salsoc/ v4n1/4n1a04.pdf

Goleman, D. (2004). La inteligencia Emocional. Barcelona: Vergara.

Gómez, O. (2006). Voces de memoria y dignidad. Modulo Aspectos Psicosociales de la Reparación Integral. Bogotá, D.C.: AFROS.

González, R., Montoya, I., Casullo, M. y Bernabéu, J. (2002). Relación entre estilos y estrategias de afrontamiento y bienestar psicológico en adolescentes. Psicothema, 14(2), 363-368.Recuperado de http://www.psicothema.com/pdf/733.pdf

González, J. y Marrero, R. (2017). Determinantes sociodemográficos y personales del bienestar subjetivo y psicológico en población mexicana. Suma psicológica, 24(1)59-66. https://doi.org/10.1016/j.sumpsi.2017.01.002

Gossaín, J. (abril 20, 2015). Las desgarradoras cifras de la violencia contra los niños. El Tiempo. [Online]. Disponible en https://www.eltiempo.com/archivo/documento/CMS15602838

Hernández, R., Fernández, C. y Baptista, P. (2010). Metodología de la investigación. 5 ed. México, D.F.: Mac Graw Hill.

Manrique, K., Turizo, Y. y Martínez, M. (2008). Estudio correlacional entre el bienestar psicológico, subjetivo y social, y el fatalismo, el trauma y las cogniciones irracionales postraumáticas, en personas adultas, desplazadas por la violencia sociopolítica, radicadas en la ciudad de Barranquilla. Tesis de grado, Universidad del Norte, Colombia. 
Matalinares, M., Díaz, G., Arenas, C., Raymundo, O., Baca, D., Uceda, J. e Yaringaño, J. (2016). Afrontamiento al estrés y bienestar psicológico en estudiantes universitarios de Lima y Huancayo. Revista IIPSI, 19(2), 123-143. https://doi.org/10.15381/rinvp. v19i2.12894

Mayordomo, T., Sales, A., Satorres, E. y Meléndez., J. (2016). Bienestar psicológico en función de la etapa de vida, el sexo y su interacción. Pensamiento Psicológico, 14(2), 101-112. https://doi.org/10.11144/Javerianacali.PPSI14-2.bpfe

Morales, M. y González, A. (2014). Resiliencia-Autoestima-Bienestar psicológico y Capacidad intelectual de estudiantes de cuarto medio de buen rendimiento de liceos vulnerables. Estudios Pedagógicos, 40(1), 215-228. http://dx.doi.org/10.4067/S071807052014000100013

Muñoz, G. y Orrego, A. (2016). Con las manos en alto” el concepto de víctima del conflicto armado: una aproximación desde la literatura. Nuevo Derecho, 12(18), 157-175. Recuperado de https://dialnet.unirioja.es/descarga/articulo/5848567.pdf

Muratori, M., Zubieta, E., Ubillos, S. y González, L. (2015). Felicidad y Bienestar Psicológico: Estudio Comparativo Entre Argentina y España. PSYKHE, 24(2), 1-18. http:// dx.doi.org/10.7764/psykhe.24.2.900

Oramas, A., Santana, S. y Vergara, A. (2006). El bienestar psicológico, un in dicador positivo de la salud mental. Revista Cubana de Salud y Trabajo, 7(1-2), 34-9. Recuperado de http://www.sld.cu/galerias/pdf/sitios/insat/rst06106.pdf

Patiño, S. (2015). Relación entre el bienestar social, el bienestar psicológico y el bienestar subjetivo en mujeres víctimas de violencia sociopolítica. Tesis Magistral. Universidad del Norte, Colombia.

Ramírez, N., Juárez, F., Parada, A., Guerrero, J., Romero, Y., Salgado, A. y Vargas, M. (2016). Afectaciones Psicológicas, Estrategias de Afrontamiento y Niveles de Resiliencia de Adultos Expuestos al Conflicto Armado en Colombia. Revista colombiana de psicología, 25(1), 125-140. http://dx.doi.org/10.15446/rcp.v25n1.49966

República de Colombia. Centro Nacional de Memoria Histórica. (2013). Los impactos y los daños causados por el conflicto armado en Colombia. En, Informe general (Capítulo IV, pp. 259-327). [Online]. Recuperado de https://reliefweb.int/sites/reliefweb.int/files/resources/capitulo\%204.pdf

República de Colombia. DPS. (1969). Programa jóvenes en acción. [Online]. Disponible en https://prosperidadsocial.gov.co/

República de Colombia. Congreso de la República. (junio 10 de 2011). Medidas de atención, asistencia y reparación integral a las víctimas del conflicto armado interno y se dictan otras disposiciones. [Ley 1448]. Diario Oficial: 48.096. Recuperado de https://www.unidadvictimas.gov.co/sites/default/files/documentosbiblioteca/ley-1448de-2011.pdf

Rodríguez, Y., Negrón. N., Maldonado, Y., Quiñones, A. y Toledo, O. (2015). Dimensiones de bienestar psicológico y apoyo social percibido con relación al sexo y nivel de estudio en universitarios. Avances en PsicologíaLatinoamericana, 33(1), 31-43. http://dx.doi. org/10.12804/apl33.01.2015.03 
Romero, L., Zapata, R., García, A., Brustad, R., Garrido, R. y Letelier, A. (2010). Estrategias de afrontamiento y bienestar psicológico en jóvenes tenistas de competición. Revista de Psicología del Deporte, 19(1), 117-133. Recuperado de https://www.rpd-online. com/article/view/660

Ryff, C. (1989a). Beyond Ponce de Leon and life satisfaction: New directionsin quest of successful aging. International Journal of Behavioral Development, 12(1), 35-55. http:// dx.doi.org/10.1177/016502548901200102

Ryff, C. (1989b). La felicidad lo es todo, ¿o no? Exploraciones sobre el significado del bienestar psicológico. Revista de personalidad y psicología social, 57(6), 1069-1081. http:// dx.doi.org/10.1037/0022-3514.57.6.1069

Ryff, C. D. \& Keyes, C. L. M. (1995). The structure of psychological well-being revisited. Journal of Personality and Social Psychology, 69(4), 719-727. http://dx.doi. org/10.1037/0022-3514.69.4.719

Ryff, C. D. \& Singer, B. H. (1998). The Contours of Positive Human Health. Psychologial Inquiry, 9(1), 1-28. http://dx.doi.org/10.1207/s15327965pli0901_1

Semana. (octubre 25, 2016). El impacto psicosocial de los niños víctimas del conflicto. semana.com. [Online]. Disponible en https://www.semana.com/educacion/articulo/impacto-del-conflictoarmado-en-ninos-de-colombia/501110

Schwartz, B. (2000). Self-Determination the tyranny of Freedom. American Pssychologist, 55(1),79-88. http://dx.doi.org/10.1037/0003-066X.55.1.79

Torres, E., Rojas, E. y Ríos, K. (2018). Bienestar psicológico de la población víctima del conflicto armado en las ciudades de Barrancabermeja, Cali, Medellín, Montería y Santa Marta. Tesis de pregrado. Universidad Cooperativa de Colombia, Barrancabermeja, Colombia. Recuperado de https://repository.ucc.edu.co/bitstream/20.500.12494/6785/3/2018_conflicto_armado_poblacion.pdf

Trejos, L. (2013). Colombia: una revisión teórica de su conflicto armado. Revista Enfoques, 11(18), 55-75. Disponible en http://www.revistaenfoques.cl/index.php/revista-uno/article/view/52

UNICEF. (2004). Desarrollo Psicosocial de los niños y las niñas. [Online]. Recuperado de https://www.oijj.org/sites/default/files/documental_5432_es.pdf

Yarlequé, L., Alva, L., Monroe, J., Núñez, E., Navarro, L., Padilla, M., Matalinares, M., Navarro, L. y Campos, J. (2016). Procrastinación, estrés y bienestar psicológico en estudiantes de educación superior de Lima yJunín. Horizonte de la Ciencia, 6(10), 173-184. Disponible en http://revistas.uncp.edu.pe/index.php/horizontedelaciencia/article/view/367

Van Dierendonck, D. (2004). The construct validity of Ryff's Scale of Psychological wellbeing and its extension with spiritual well-being. Personality and Individual Differences, 36(3), 629-644. https:// https://doi.org/10.1016/S0191-8869(03)00122-3

Veenhoven, R. (1994). El sentido de la satisfacción con la vida. Intervención Psicosocial, 3(9), 87-116. Disponible en http://www.copmadrid.org/webcopm/publicaciones/social/1994/ vol3/arti8.htm

Véliz, A. (2012). Propiedades psicométricas de la Escala deBienestar Psicológico y estructura factorial en universitarios chilenos. Psicoperspectivas, 11(2), 143-163. http://dx.doi. org/10.5027/psicoperspectivas-Vol11-Issue2-fulltext-196 
Viñas, F., González, M., García, Y., Malo, S. y Casas, F. (2015). Los estilos y estrategias de afrontamiento y su relación con el bienestar personal en una muestra de adolescentes. Anales de psicología, 31(1), 226-233. http://dx.doi.org/10.6018/analesps.31.1.163681

Claudina Esther Anaya Garcia es Psicóloga con máster en Psicología Forense del Instituto Superior de Estudios Psicológicos (ISEP, España). Con experiencia en el área clínica y social en atención a poblaciones vulnerables. Coordinadora del proyecto MAM del Centro Cultural Jáfana Jáfana, en atención psicosocial a mujeres migrantes venezolanas en condiciones de mendicidad en la ciudad de Santa Marta. https://orcid.org/0000-0001$6317-9843$

Linda Carolina Sánchez Rende es Psicóloga con magíster en desarrollo integral de niños y adolescentes de la Universidad Cooperativa de Colombia. Se ha desempeñado en el área clínica y social en la atención de poblaciones vulnerables. https://orcid.org/00000003-2565-0950

Amanda Miguel Iguarán Jiménez es Magíster en Educación de la Universidad de la Costa, CUC (colombia). Especialista en docencia Universitaria y psicóloga de la Universidad del Magdalena, docente catedrática e intergrante del grupo de Investigación Cognición y Educación del programa de Psicología de la Universidad del Magdalena. https:/orcid. org/0000-0001-6317-9843 